\title{
Associations Between Trajectories of Leisure-Time Physical Activity and Television Viewing Time Across Adulthood: The Cardiovascular Risk in Young Finns Study
}

\author{
Xiaolin Yang, Irinja Lounassalo, Anna Kankaanpää, Mirja Hirvensalo, Suvi P. Rovio, \\ Asko Tolvanen, Stuart J.H. Biddle, Harri Helajärvi, Sanna H. Palomäki, \\ Kasper Salin, Nina Hutri-Kähönen, Olli T. Raitakari, and Tuija H. Tammelin
}

\begin{abstract}
Background: The purpose of this study was to examine trajectories of leisure-time physical activity (LTPA) and televisionviewing (TV) time and their associations in adults over 10 years. Methods: The sample comprised 2934 participants (men, $46.0 \%$ ) aged 24-39 years in 2001 and they were followed up for 10 years. LTPA and TV time were assessed using self-report questionnaires in 2001, 2007, and 2011. Longitudinal LTPA and TV-time trajectories and their interactions were analyzed with mixture modeling. Results: Three LTPA (persistently highly active, $15.8 \%$; persistently moderately active, $60.8 \%$; and persistently low active, $23.5 \%$ ) and 4 TV time (consistently low, 38.6\%; consistently moderate, 48.2\%; consistently high, $11.7 \%$; and consistently very high, $1.5 \%$ ) trajectory classes were identified. Persistently highly active women had a lower probability of consistently high TV time than persistently low-active women $(P=.02)$, whereas men who were persistently highly active had a higher probability of consistently moderate TV time and a lower probability of consistently low TV time than their persistently low-active counterparts $(P=.03$ and $P=.01$, respectively). Conclusions: Maintaining high LTPA levels were accompanied by less TV over time in women, but not in men. The associations were partially explained by education, body mass index, and smoking.
\end{abstract}

Keywords: exercise, sedentary behavior, screen time, epidemiology

In recent decades, lifestyle has become more sedentary both during working hours and leisure time, especially in high-income countries. ${ }^{1}$ Sedentary behavior is commonly defined as any waking behavior characterized by an energy expenditure $\leq 1.5$ metabolic equivalents while in a sitting, lying, or reclining posture, ${ }^{2}$ and it should be distinguished from "physical inactivity."1 Of various sedentary behaviors, television viewing (TV) time still remains the most prevalent in Finland despite the proliferation of other electronic devices. ${ }^{3}$ Increased TV time has been found to be associated with more adverse health and behavioral outcomes than other domains of sedentary behavior (eg, using a computer, sitting during transportation, or sitting at work), ${ }^{4,5}$ and it may even lead to premature deaths during adulthood. ${ }^{6}$ On the other hand, evidence shows that regular leisure-time physical activity (LTPA) has longterm health benefits and contributes substantially to reduction of all-cause mortality. ${ }^{7}$

\footnotetext{
Yang and Lounassalo contributed equally to this work. Yang, Kankaanpää, and Tammelin are with the LIKES Research Centre for Physical Activity and Health, Jyväskylä, Finland. Lounassalo, Hirvensalo, Palomäki, and Salin are with the Faculty of Sport and Health Sciences, University of Jyväskylä, Jyväskylä, Finland. Rovio and Raitakari are with the Research Centre of Applied and Preventive Cardiovascular Medicine, University of Turku and Department of Clinical Physiology, Turku, Finland; and Nuclear Medicine, Turku University Hospital, Turku, Finland. Tolvanen is with the Methodology Center for Human Sciences, University of Jyväskylä, Jyväskylä, Finland. Biddle is with the Institute for Resilient Regions, University of Southern Queensland, Springfield, QLD, Australia. Helajärvi is with the Departments of Physiology and Health and Physical Activity, Paavo Nurmi Centre, University of Turku, Turku, Finland. Hutri-Kähönen is with the Department of Pediatrics, Tampere University Hospital, University of Tampere, Tampere, Finland. Yang (xiaolin.yang@likes.fi) is corresponding author.
}

Although LTPA is one key intervention to reduce TV time, the association between LTPA and TV time in adults remains to be clarified. According to a recent systematic review, ${ }^{8}$ only 3 observational studies ( 2 cross-sectional ${ }^{9,10}$ and 1 longitudinal ${ }^{11}$ ) report a small or moderate inverse association between the 2 variables. The review concluded that sedentary behavior does not displace moderate to vigorous physical activity (MVPA) but more likely replaces light-intensity physical activity when using objective monitoring devices. ${ }^{8}$ Regarding associations between physical activity and TVtime trajectories, only 2 longitudinal studies have explored such an association in youth. ${ }^{12,13}$ In both of these studies, participants who maintained higher MVPA levels decreased their TV time. Thus, the developmental pathways of high MVPA and low TV time could be related. However, there remains a lack of longitudinal research exploring the linkages between LTPA and TV-time trajectories in adulthood. The strength of the trajectory modeling is that rather than assuming the existence of distinct subgroups (ie, trajectories) in a population, it identifies them based on the population data. ${ }^{14}$

The aim of this study was threefold: (1) to describe developmental trajectory subgroups of both LTPA and TV time from young adulthood to early midlife over a 10-year follow-up period, (2) to identify the linkages between LTPA and TV-time trajectory classes, and (3) to examine gender differences in such associations with taking into account age, education, body mass index (BMI), and smoking.

\section{Methods}

\section{Participants}

The Cardiovascular Risk in Young Finns Study is an ongoing longitudinal population-based study consisting of 6 cohorts born in 
$1962,1965,1968,1971,1974$, and 1977. The sample of healthy children and adolescents, aged 3, 6, 9, 12, 15, and 18 years, were randomly selected in 1980 from the 5 Finnish university cities with medical schools (Helsinki, Kuopio, Oulu, Tampere, and Turku) and their surrounding communities $(\mathrm{N}=3596,83.0 \%$ of those who were first invited). The representativeness of study participants has been tested in 2001 by comparing their baseline (1980) characteristics to subjects lost to follow-up. ${ }^{15}$ The results showed participants to be older and more often women than subjects lost to follow-up. However, no significant differences were observed in LTPA or TV time between participants and dropouts. ${ }^{16,17}$ The detailed description of the Young Finns Study, reasons for nonparticipation at follow-ups, and the characteristics of the participants have been reported elsewhere. ${ }^{15}$

For the present study, we chose 2001 as the baseline because that was the year when the self-reported TV time was collected for the first time from all 6 cohorts. The participants were aged 24-39 years in 2001, and hence, 34-49 years in 2011. Those with missing information on both LTPA and TV-time variables were excluded. Complete data on all variables were available for 2934 healthy adults (men, 46.0\%). The study protocol was reviewed and approved by the ethics committees of each of the 5 participating universities. The informed consent of all subjects was obtained in accordance with the Helsinki Declaration. ${ }^{15}$

\section{Leisure-Time Physical Activity}

LTPA in 2001, 2007, and 2011 was measured by a short self-report questionnaire. The questions consisted of items on the intensity of LTPA, frequency of vigorous LTPA, hours spent on vigorous LTPA, average duration of an LTPA session, and participation in organized LTPA. All items were first recoded (1 = inactivity or very low activity to $3=$ regular or vigorous activity) and then summed to create a physical activity index ranging from 5 to $15,{ }^{17}$ with high scores indicative of higher levels of LTPA. Testretest reliability coefficients of the LTPA values between 2001 and 2007 were $>.60 .{ }^{17}$ The validity of the LTPA values has been tested by showing a statistically significant correlation with the indicators of exercise capacity (hypothetical maximal workload sustainable for $6 \mathrm{~min})$ in a subsample for women $(r=.49, P<.001)$ and men $(r=.53, P<.001)^{18}$ and with 7 -day pedometer data obtained for total steps $(r=.24, P<.001)$ and aerobic steps $(r=.31, P<.001) .{ }^{19}$

\section{TV Time}

Self-reported TV time in adulthood was measured with a question: "How many hours/minutes on average per day do you spend watching television?" 16,20 Daily TV time was recorded in minutes in 2001 and in hours in 2007. In 2011, daily TV time was measured in minutes separately for weekdays and weekend days. A mean daily TV time was calculated $([5 \times$ weekday $]+[2 \times$ weekend $]) / 7$. To have the same unit of measurement for TV time, all 3 measurements of daily TV time were converted into 1-hour increments (hours of daily TV time) prior to statistical analysis.

\section{Confounders}

In 2001, educational attainment was self-reported and measured as completed school years. Body weight was measured with a Seca scale (Vogel \& Halke, Hamburg, Germany) and body height with a Seca anthropometer (Vogel \& Halke). BMI was calculated as weight $(\mathrm{kg}) /$ height $\left(\mathrm{m}^{2}\right)$. Smoking habits were obtained by a questionnaire, those participants smoking on a daily basis were deemed as smokers.

\section{Statistical Analysis}

Descriptive statistics were calculated using IBM SPSS Statistics for Windows, version 20.0 (IBM Corp, Armonk, NY) and further modeling was performed using Mplus, version 7.0 (Los Angeles, CA). ${ }^{21}$ To identify LTPA and TV-time trajectory subgroups in adulthood, latent profile analyses were conducted. Latent profile analysis is a special case of a wider family of mixture models. The heterogeneous population is considered to consist of subgroups of individuals, but the group membership is unknown. Mixture modeling is a tool to statistically identify these homogeneous subgroups in a data-driven way. First, the latent profile analysis was carried out separately for both outcomes. The classification was based on the means of the outcome measures in 2001, 2007, and 2011, and error variances were assumed to be equal across classes. A model with 2 to 6 classes was fitted with gender and age covariates. Several fit indices were used to evaluate the goodness of fit of the latent profile analyses with different number of classes: Akaike's information criterion, Bayesian information criterion, and sample-size adjusted Bayesian information criterion. The model with lower values of information criteria fitted the data better than an alternative model with higher values. Furthermore, the following statistical tests were used to determine the sufficient number of classes: Vuong-LoMendell-Rubin likelihood ratio test (VLMR), Lo-Mendell-Rubin (LMR) adjusted likelihood ratio test, and parametric bootstrapped likelihood ratio test. The estimated model was compared to the model with one class less, and the low $P$ value of the test indicates that the model with one class less was rejected in favor of the estimated model. The quality of the classification was evaluated using entropy values and the average posterior probabilities for most likely latent class membership (all ranging from 0 to 1 for both measures, value 1 indicating perfect classification). The average posterior probabilities higher than 0.7 were considered acceptable. ${ }^{21}$

Second, interrelationship between the longitudinal patterns of LTPA and TV time was examined via transition probabilities obtained from multinomial logistic regression analysis (ie, dual trajectory model). ${ }^{22} \mathrm{~A}$ multinomial logistic regression model was specified between the latent class variables: the latent class variable of TV time was regressed on the latent class variable of LTPA. Gender was allowed to be associated with both the latent class variables and to moderate the association between LTPA and TV time. ${ }^{19}$ The model was adjusted for potential confounding variables including age, education, BMI, and smoking. The confounders were allowed to be associated with both the latent class variables. The differences in the gender effect on latent class variable of TV time across the LTPA classes (ie, interaction of gender and LTPA on TV time) were tested. The adjusted transition probabilities (ie, conditional probabilities for TV-time trajectory classes given to LTPA trajectory classes) were calculated separately for men and women using the parameter estimates of the model and setting age, education, and BMI to their overall mean and smoker to nonsmoker.

Missing data were assumed to be missing at random. Parameters of the models were estimated by using the full information maximum likelihood (FIML) method with robust SEs, which enabled to use all the data available. The FIML method produced unbiased parameter estimates under the missing at random assumption.

\section{Results}

Participants (1350 men and 1584 women) having at least one measure of LTPA or TV time were included in the study. For 
LTPA, 1431 participants (48.8\%) completed all 3 measurements, $775(26.4 \%)$ completed 2, $675(23.0 \%)$ completed 1 , and $53(1.8 \%)$ did not have any information on LTPA. For TV time, the corresponding figures were 1566 (53.4\%), 727 (24.8\%), 636 (21.7\%), and $5(0.2 \%)$ participants, respectively. Descriptive characteristics of the study sample are presented in Table 1.

Adjusted models (adjusted for gender and age) with 4 classes provided the best fit for the LTPA data (Table 2). VLMR and LMR were significant $(P<.001$ for both) for the models with $<5$ classes. Model fit of the models for LTPA improved with each step. However, after a 4-class solution, some of the average posterior probabilities dropped under an acceptable level and, therefore, a 4-class solution was considered optimal. Longitudinal LTPA trajectory classes were identified: persistently very highly active $(3.8 \%)$, persistently highly active $(17.5 \%)$, persistently moderately

\section{Table 1 Characteristics of the Study Sample}

\begin{tabular}{lcc}
\hline Variables & $\mathbf{n}$ & Mean (SD)/\% of total $\mathbf{n}$ \\
\hline TV time in 2001, h/d & 2594 & $2.0(1.1)$ \\
TV time in 2007, h/d & 2224 & $1.7(1.1)$ \\
TV time in 2011, h/d & 1970 & $1.8(1.2)$ \\
LTPA in 2001, score & 2453 & $8.8(1.9)$ \\
LTPA in 2007, score & 2173 & $8.8(1.8)$ \\
LTPA in 2011, score & 1924 & $9.0(1.8)$ \\
Age (y) in 2001 & 2934 & $31.4(4.9)$ \\
Gender, \% & 2934 & \\
Men & 1350 & 46.0 \\
Women & 1584 & 54.0 \\
Education (y) in 2001 & 2604 & $14.5(3.1)$ \\
Body mass index in 2001 & 1924 & $25.0(4.4)$ \\
Smoking in 2001, \% & 2547 & 29.6 \\
Smoker & 754 & 70.4 \\
Nonsmoker & 1793 & \\
\hline
\end{tabular}

Abbreviations: LTPA, leisure-time physical activity; TV, television viewing. active $(51.1 \%)$, and persistently low active $(27.6 \%)$. To avoid small-cell frequencies, the 3-class solution for LTPA (persistently highly active, $15.8 \%$; persistently moderately active, $60.8 \%$; and persistently low active, $23.5 \%$ ) was considered more appropriate than a 4-class solution for further analyses.

Model fit of the adjusted models (adjusted for gender and age) for TV time improved with each step (Table 2). Because only small additional classes were extracted from the 5-class solution forward (class sizes $<5 \%$ ), the 4-class solution was considered optimal. Longitudinal TV-time trajectory classes were identified: consistently low TV time $(\leq 1 \mathrm{~h} / \mathrm{d}, 38.6 \%)$, consistently moderate TV time $(2 \mathrm{~h} / \mathrm{d}, 48.2 \%)$, consistently high TV time ( $\geq 3 \mathrm{~h} / \mathrm{d}, 11.7 \%)$, and consistently very high TV time ( $\geq 5 \mathrm{~h} / \mathrm{d}, 1.5 \%)$. The last 2 categories were combined to form "high" in order to further analyze an interpretable model. Quality of the classification for both LTPA and TV time was acceptable.

The estimation results of the multinomial logistic regression between latent classes of LTPA and TV time and moderating effect of gender are shown in Table 3. Participants in both persistently moderately active and highly active classes had a lower probability of consistently high TV time than those in the persistently lowactive class (unstandardized regression coefficient $b=-1.14, \mathrm{SE}=$ $0.35, P=.001$ and $b=-2.35, \mathrm{SE}=0.84, P=.005$, respectively). These associations disappeared between the persistently moderately active and low-active classes $(b=-0.60, \mathrm{SE}=0.37, P=.11)$ and attenuated between the persistently highly active and low-active classes $(b=-1.44, \mathrm{SE}=0.70, P=.04)$ after additional adjustment for education, BMI, and smoking. Gender effects on latent class variable of TV time differed across the LTPA classes. The gender (men) effect on the consistently high TV time class was positive within the persistently highly active $(b=1.98, \mathrm{SE}=0.90, P=.03)$ and moderately active $(b=0.83, \mathrm{SE}=0.26, P=.001)$ classes. The male gender effect on the consistently moderate TV time was also positive within the persistently highly active class $(b=0.96, \mathrm{SE}=$ $0.38, P=.01)$. All associations concerning gender effects disappeared after adjustment for the confounding variables.

The age-adjusted latent transition probabilities between LTPA and TV-time trajectory classes for men and women are illustrated in Figure 1A and 1B. The probability of the consistently high TV time

Table 2 The Estimation Results of Latent Profile Analyses of LTPA $(n=2886)$ and TV Time $(n=2929)$

\begin{tabular}{ccccccccc}
\hline Class & AIC & BIC & ABIC & VLMR & LMR & BLRT & Entropy & Class sizes $^{\mathbf{a}, \mathbf{b}}$ \\
\hline LTPA & & & & & & & & \\
2 & 26,072 & 26,143 & 26,105 & $<.001$ & $<.001$ & $<.001$ & 0.52 & $58.5 \%, 41.4 \%$ \\
3 & 25,707 & 25,815 & 25,757 & $<.001$ & $<.001$ & $<.001$ & 0.62 & $60.8 \%, 23.5 \%, 15.8 \%$ \\
4 & 25,612 & 25,755 & 25,679 & $<.001$ & $<.001$ & $<.001$ & 0.62 & $51.1 \%, 27.6 \%, 17.5 \%, 3.8 \%$ \\
5 & 25,570 & 25,749 & 25,654 & .03 & .03 & $<.001$ & 0.62 & $50.8 \%, 23.4 \%, 16.9 \%, 5.4 \%, 3.5 \%$ \\
6 & 25,547 & 25,761 & 25,647 & .32 & .33 & $<.001$ & 0.64 & $48.5 \%, 22.7 \%, 17.5 \%, 4.7 \%, 3.4 \%, 3.1 \%$ \\
TV time & & & & & & & & $83.4 \%, 16.6 \%$ \\
2 & 20,058 & 20,130 & 20,092 & $<.001$ & $<.001$ & $<.001$ & 0.78 & $62.2 \%, 34.6 \%, 3.2 \%$ \\
3 & 19,553 & 19,660 & 19,603 & .20 & .21 & $<.001$ & 0.70 & $48.2 \%, 38.6 \%, 11.7 \%, 1.5 \%$ \\
4 & 19,335 & 19,479 & 19,402 & .47 & .47 & $<.001$ & 0.67 & $41.8 \%, 38.2 \%, 17.0 \%, 2.4 \%, 0.6 \%$ \\
5 & 19,026 & 19,206 & 19,111 & .04 & .05 & $<.001$ & 0.84 & .001 \\
6 & 18,856 & 19,071 & 18,957 & .22 & .22 & $<.001$ & 0.85 & $41.8 \%, 37.8 \%, 17.0,2.4 \%, 0.6 \%, 0.4 \%$ \\
\hline
\end{tabular}

Abbreviations: ABIC, sample-size adjusted Bayesian information criterion; AIC, Akaike's information criterion; BLRT, parametric bootstrapped likelihood ratio test; LMR, Lo-Mendell-Rubin adjusted likelihood ratio test; LTPA, leisure-time physical activity; TV, television viewing; VLMR, Vuong-Lo-Mendell-Rubin likelihood ratio test.

${ }^{\mathrm{a} C}$ Class counts and proportions for the latent class patterns. ${ }^{\mathrm{b}} \mathrm{Gender}$ and age were included to predict group membership. 
Table 3 Parameter Estimates for the Relationship Between LTPA and TV Time and Modifying Effect of Gender $(n=2934)$

\begin{tabular}{|c|c|c|c|c|c|c|}
\hline \multirow[b]{3}{*}{ Group } & \multicolumn{6}{|c|}{ TV-time class (consistently low as reference) } \\
\hline & \multicolumn{3}{|c|}{ Consistently moderate } & \multicolumn{3}{|c|}{ Consistently high } \\
\hline & b & SE & $\boldsymbol{P}$ & b & SE & $\boldsymbol{P}$ \\
\hline \multicolumn{7}{|l|}{ LTPA class } \\
\hline \multicolumn{7}{|l|}{ Model 1} \\
\hline \multicolumn{7}{|c|}{ Persistently low active (as reference) } \\
\hline Persistently moderately active & -0.22 & 0.33 & .50 & -1.14 & 0.35 & .001 \\
\hline Persistently highly active & -0.59 & 0.39 & .14 & -2.35 & 0.84 & .01 \\
\hline \multicolumn{7}{|l|}{ Model 2} \\
\hline \multicolumn{7}{|c|}{ Persistently low active (as reference) } \\
\hline Persistently moderately active & 0.07 & 0.34 & .77 & -0.60 & 0.37 & .11 \\
\hline Persistently highly active & -0.12 & 0.40 & .83 & -1.44 & 0.70 & .04 \\
\hline \multicolumn{7}{|c|}{ Gender effect (male) on TV time in distinct LTPA classes } \\
\hline \multicolumn{7}{|l|}{ Model 1} \\
\hline Persistently low active & -0.31 & 0.37 & .40 & -0.32 & 0.41 & .44 \\
\hline Persistently moderately active & 0.31 & 0.20 & .12 & 0.83 & 0.26 & .001 \\
\hline Persistently highly active & 0.96 & 0.38 & .01 & 1.98 & 0.90 & .03 \\
\hline \multicolumn{7}{|l|}{ Model 2} \\
\hline Persistently low active & -0.43 & 0.38 & .26 & -0.71 & 0.39 & .07 \\
\hline Persistently moderately active & 0.10 & 0.21 & .64 & 0.49 & 0.26 & .06 \\
\hline Persistently highly active & 0.70 & 0.39 & .07 & 1.41 & 0.74 & .06 \\
\hline
\end{tabular}

Abbreviations: $b$, regression coefficient; LTPA, leisure-time physical activity; TV, television viewing. Note: The consistently very high TV-time class that constituted $<5 \%$ of the sample was omitted from the table. Model 1 was adjusted for age. Model 2 was additionally adjusted for education, body mass index, and smoking.
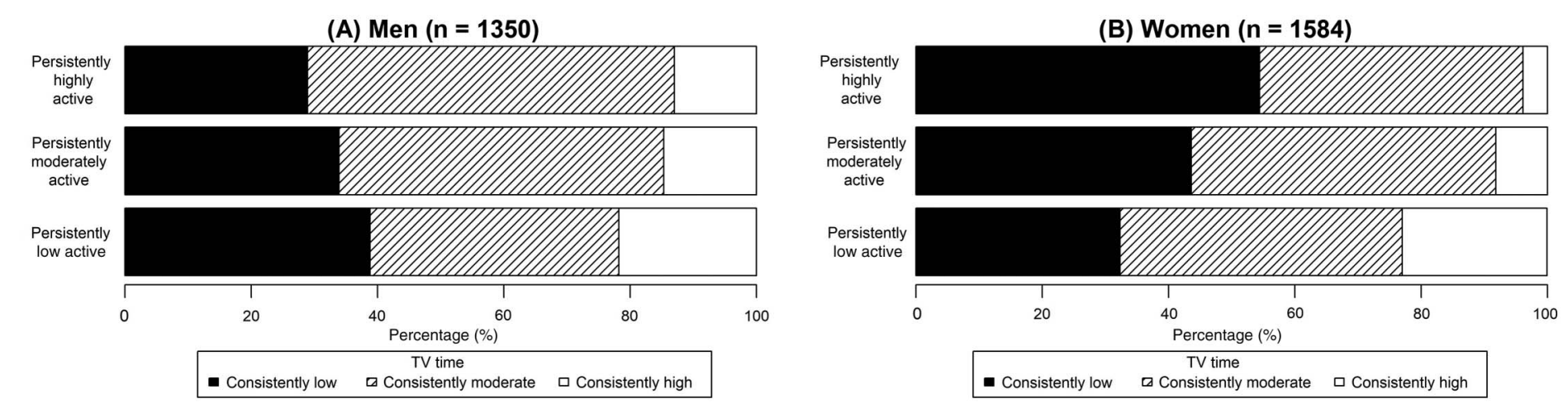

Figure 1 - Latent transition probabilities of television viewing time trajectories conditional to leisure-time physical activity trajectories adjusted for age for men (A) and women (B).

was lower in persistently high-active women than in persistently low-active women (3.9\% vs 22.9\%, $P<.001$; Figure 1B). Among men, a similar tendency was observed but the difference between the classes was only marginally significant $(13.0 \%$ vs $21.8 \%$, $P=.06$; Figure 1A). In addition, the probability of the consistently low TV time was higher in persistently high-active women than in persistently low-active ones $(54.4 \%$ vs $32.3 \% ; P=.01)$, whereas no such difference was observed in men $(28.9 \%$ vs $38.8 \%, P=.13)$.

After additional adjustment for BMI, education, and smoking, the associations between the persistently highly active and lowactive classes on the consistently high TV time attenuated in women (9.2\% vs $26.2 \%, P=.02)$ and disappeared in men (17.0\% vs $19.8 \%$, $P=.57)$. Men who were persistently highly active had a higher level of consistently moderate TV time than those who were persistently low active $(68.4 \%$ vs $51.1 \%$; $P=.03)$, whereas persistently highactive men had a lower level of consistently low TV time than their persistently low-active counterparts $(14.2 \%$ vs $29.3 \% ; P=.01)$. No group differences were observed in either consistently moderate TV-time class or consistently low TV-time class in women after additional adjustment for the covariates.

\section{Discussion}

The purpose of this study was to identify distinctive, potentially previously unobserved, stable, and changing LTPA and TV-time trajectories among Finnish men and women over a period of 10 years; 
and to investigate how the identified LTPA trajectory classes were related to the TV-time trajectory classes. Three LTPA (persistently highly active, persistently moderately active and persistently low active) and 4 TV-time (consistently low, consistently moderate, consistently high, and consistently very high) trajectory classes were identified. We found an inverse association between persistently high LTPA and excessive TV time in women, but not in men. The differences were partially explained by education, BMI, and smoking.

The largest proportion of participants was identified in the persistently moderately active class. Even though, worldwide, physical inactivity is usually more prevalent among women than among men, it is not the case in Finland. ${ }^{23}$ The present study supports this observation with the proportion of physically lowactive women being lower than physically low-active men. This study did not identify LTPA trajectory classes describing change in the LTPA behavior in adulthood, whereas previous studies have either found stable LTPA trajectory classes alone ${ }^{24}$ or both increasing and decreasing classes in addition to stable classes. ${ }^{25}$ The inconsistent findings may be due to a wide range of ages or differences in methodology or measurements. ${ }^{26}$

The consistently moderate TV-time ( $2 \mathrm{~h} / \mathrm{d}$ ) class was found to be the most prevalent (48.3\%), which slightly differs with the previous result showing that the mean daily TV time in 2015 was 2 hours and 54 minutes per day for Finnish adults aged 25-44 years. ${ }^{27}$ Our study found a smaller proportion of adults in the consistently high TV-time class and a larger proportion of adults in the consistently low TVtime class compared with the previous trajectory studies examining youth only. ${ }^{12,13,28}$ One explanation might be that adolescents have more leisure time and fewer responsibilities when compared with adults and therefore they simply spend more time watching television. Previous trajectory studies have identified TV-time change, indicating that the TV behavior has not yet become stable in youth, ${ }^{12,28}$ whereas our results suggest that the TV behavior stabilizes to a certain level during adulthood.

Few previous studies have examined the relationship between physical activity and TV-time or sedentary behavior in either men or women. A significant negative association has been found between watching television on a week day and high activity but only in men. ${ }^{29}$ In contrast, a few previous studies have reported TV time to be inversely associated with physical activity among women. ${ }^{28,30}$ We found that persistently active women spent less time watching television than persistently active men when compared with their low-active counterparts, suggesting that the amount of time women spend watching television competes with time spend on LTPA. Meanwhile, excessive TV time can coexist for men at low, moderate, or high LTPA level. This supports previous findings that sedentary behavior may be independent of MVPA levels, ${ }^{8}$ and sedentary behavior and physical activity cannot be seen only as functional opposites. ${ }^{1,29}$ In fact, it has been argued that less TV time can potentially be an important target to promote more active lifestyle for women but not for men due to these different TV-time and LTPA patterns between genders. ${ }^{30}$

One possible explanation for gender differences in the association between LTPA and TV time may be that women experience clusters of multiple health behaviors more often than men. ${ }^{31}$ Additionally, men and women have different motivations for participation in LTPA: women have more extrinsic orientation (appearance and physical condition), whereas men have more intrinsic orientation (mastery and competition). ${ }^{32}$ Thus, women's health consciousness may have an additive effect on their decision making process in TV time. Another possible explanation for these differences may be related to the use of leisure time in Finland. Finnish women spend almost an hour more on household work than men on an average day which may lead to those devoting more time to LTPA having less time for watching television. Future research may investigate the motives for TV time by gender to verify whether health-related reasons or the use of leisure time affect the decision making.

According to the crude analysis, the findings were as expected: participants who were persistently low active were more likely to watch more television than those who were persistently moderately active or highly active. However, it is noteworthy that these significant associations mainly disappeared after adjustment for education, BMI, and smoking. Findings also indicated that the relationship between LTPA and TV time for both genders was affected by the confounding variables. Thus, it cannot be excluded that relation between these 2 variables is caused by a third factor. The causality may be bidirectional: persons with higher levels of education, lower BMI, and nonsmoking ${ }^{33}$ may be more likely to participate and persist in LTPA, which, in turn, improves resources to reduce the amount of TV time. On the other hand, it is possible that each of these factors may explain directly or indirectly the reduction of TV time among women who engage in regular LTPA.

The relationship between TV time and physical activity is also complicated in the light of their joint effect on health. Evidence shows that MVPA may eliminate the increased risk of death associated with high total sitting time, and attenuates the risk associated with high TV time. ${ }^{34}$ On the other hand, even if adults meet the public health guidelines for physical activity, but also sit for longer periods of time without breaks, their metabolic health may be compromised. ${ }^{35}$ In our study, the consistently very high TV-time class accounted for only a small portion of the sample $(1.5 \%)$, but it is potentially important, since these subjects are characterized by high sitting time. Thus, future studies should seek to replicate the results in health domains.

\section{Strengths and Limitations}

To our knowledge, this was the first study to identify TV-time trajectories from young adulthood to middle age and to study their association with LTPA trajectories. Our study has several strengths, including the long follow-up time, large sample size consisting of 6 age cohorts, and recruitment of subjects throughout Finland. However, a few limitations should be acknowledged. LTPA and TV time were self-reported and measured only in leisure time and no other sedentary behavior types apart from TV time were considered. The findings are based on the data in the genetically homogeneous Finnish adults and may not generalize well to other populations, especially those from low-income countries or different ethnic groups.

The statistical analyses used for identifying trajectories have certain strengths. Since the association between LTPA and TV time was modeled via latent profile analysis, the uncertainty in class membership was taken into account in the analysis. Another strength is that trajectory modeling is data driven, meaning that it is based on objective model fit indicators for identifying the optimal number of latent classes. ${ }^{36}$ However, the selection of the number of classes was partly based on interpretability and class sizes because the latent profile analysis with a 4-class solution for LTPA could not be conducted due to the small class size of very highly active participants. This led to the selection of the 3-class solution for LTPA, which might be a source of bias: the proportion of participants reporting moderate levels of LTPA increased, 
whereas participation levels on longitudinal changes in LTPA attenuated or disappeared. This is similar to the situation in TV time, where only very few participants reported changes in TV time. Although these results may seem surprising, it is essential that some participants may increase or decrease their LTPA or TV time 10 years later, but their original behaviors have not changed enough to move toward another trajectory. The limitation of trajectory modeling is that no participant perfectly follows the identified trajectories: each trajectory is a mean description of the behavior of the subgroup, where individuals behave as similar as possible within the subgroup while differing from the other subgroups. ${ }^{37}$

\section{Conclusions}

Our study represents relatively stable LTPA and TV-time trajectory classes in adults after 10 years of follow-up. The inverse association between persistently high LTPA and excessive TV time was observed only in women after adjustment for education, BMI, and smoking. We suggest that maintaining high level of LTPA is accompanied by less TV over time for women but not men. Future studies should confirm these findings with objective monitoring devices, and the predictors, correlates and health outcomes of the class memberships should be taken into account.

\section{Acknowledgments}

The authors thank Emeritus Professor Risto Telama for giving his input on the interpretation of the results and Pinja Pesonen and Harto Hakonen for assisting with the statistical analyses and figures. The Young Finns Study has been financially supported by the Academy of Finland (grants 273971, 134309 [Eye], 126925, 121584, 124282, 129378 [Salve], 117787 [Gendi], and 41071 [Skidi]), the Ministry of Education and Culture, the Social Insurance Institution of Finland, the Special Federal Grants for University Hospitals, Kuopio, Tampere and Turku University Hospital Medical Funds, the Juho Vainio Foundation, the Paavo Nurmi Foundation, the Finnish Foundation for Cardiovascular Research, the Finnish Cultural Foundation, the Tampere Tuberculosis Foundation, the Orion-Farmos Research Foundation, the Sigrid Juselius Foundation, the Emil Aaltonen Foundation, and the Yrjö Jahnsson Foundation.

\section{References}

1. Owen N, Healy GH, Matthews CE, Dunstan DW. Too much sitting: the population-health science of sedentary behavior. Exerc Sport Sci Rev. 2010;38(3):105-113. PubMed ID: 20577058 doi:10.1097/JES. 0b013e3181e373a2

2. Tremblay MS, Aubert S, Barnes JD, et al. Sedentary Behavior Research Network (SBRN)-Terminology Consensus Project process and outcome. Int J Behav Nutr Phys Act. 2017;14:75. PubMed ID: 28599680 doi:10.1186/s12966-017-0525-8

3. Sandell L. Television viewing in Finland 2016. Finnpanel: Tennisplatsi. 2017;12. https://www.finnpanel.fi â $€^{\circ}$ lataukset â $€^{\circ}$ tv_year_2017. Accessed February 7, 2017.

4. Basterra-Gortari FJ, Bes-Rastrollo M, Gea A, Nunez-Cordoba JM, Toledo E, Martinez-Gonzalez MA. Television viewing, computer use, time driving and all-cause mortality: the SUN cohort. J Am Heart Assoc. 2014;3(3):e000864. PubMed ID: 24965030 doi:10.1161/ JAHA.114.000864

5. Kim Y, Wilkens LR, Park S-Y, Goodman MT, Monroe KR, Kolonel LN. Association between various sedentary behaviours and all-cause, cardiovascular disease and cancer mortality: the Multiethnic Cohort
Study. Int J Epidemiol. 2013;42(4):1040-1056. PubMed ID: 24062293 doi:10.1093/ije/dyt108

6. Keadle SK, Moore SC, Sampson JN, Xiao Q, Albanes D, Matthews CE. Causes of death associated with prolonged TV viewing: NIHAARP diet and health study. Am J Prev Med. 2015;49(6):811-821. PubMed ID: 26215832 doi:10.1016/j.amepre.2015.05.023

7. Reiner M, Niermann C, Jekauc D, Woll A. Long-term health benefits of physical activity - a systematic review of longitudinal studies. BMC Public Health. 2013;13:813. PubMed ID: 24010994 doi:10. 1186/1471-2458-13-813

8. Mansoubi M, Pearson N, Biddle SJH, Clemes S. The relationship between sedentary behaviour and physical activity in adults: a systematic review. Prev Med. 2014;69:28-35. doi:10.1016/j.ypmed. 2014.08.028

9. Sugiyama T, Salmon J, Dunstan DW, Bauman AE, Owen N. Neighborhood walkability and TV viewing time among Australian adults. Am J Prev Med. 2007;33(6):444-449. PubMed ID: 18022059 doi:10.1016/j.amepre.2007.07.035

10. Sugiyama T, Healy GN, Dunstan DW, Salmon J, Owen N. Joint associations of multiple leisure-time sedentary behaviours and physical activity with obesity in Australian adults. Int J Behav Nutr Phys Act. 2008;5:35. PubMed ID: 18590570 doi:10.1186/ 1479-5868-5-35

11. Lakerveld J, Dunstan D, Bot S, et al. Abdominal obesity, TV-viewing time and prospective declines in physical activity. Prev Med. 2011;53:299-302. doi:10.1016/j.ypmed.2011.07.012

12. Kwon S, Janz KF, Letuchy EM, Burns TL, Levy SM. Developmental trajectories of physical activity, sports, and television viewing during childhood to young adulthood: Iowa Bone Development Study. JAMA Pediatr. 2015;169(7):666-672. PubMed ID: 25984811 doi:10.1001/jamapediatrics.2015.0327

13. Kwon S, Lee J, Carnethon MR. Developmental trajectories of physical activity and television viewing during adolescence among girls: National Growth and Health Cohort Study. BMC Public Health. 2015;15(667):1-9. doi:10.1186/s12889-015-2043-4

14. Nagin DS. Group-Based Modeling of Development. Cambridge, MA: Harvard University Press; 2005.

15. Raitakari OT, Juonala M, Rönnemaa T, et al. Cohort profile: the cardiovascular risk in Young Finns Study. Int J Epidemiol. 2008; 37(6):1220-1226. PubMed ID: 18263651 doi:10.1093/ije/dym225

16. Yang X, Kankaanpää A, Biddle SJH, et al. Tracking of television viewing time during adulthood: the Young Finns Study. Med Sci Sports Exerc. 2017;49(1):71-77. PubMed ID: 27540995 doi:10. 1249/MSS.0000000000001072

17. Telama R, Yang X, Leskinen E, et al. Tracking of physical activity from early childhood through youth into adulthood. Med Sci Sport Exerc. 2014;46(5):955-962. doi:10.1249/MSS.0000000000000181

18. Telama R, Yang X, Viikari J, Välimäki I, Wanne O, Raitakari O. Physical activity from childhood to adulthood: a 21-year tracking study. Am J Prev Med. 2005;28(3):267-273. PubMed ID: 15766614 doi:10.1016/j.amepre.2004.12.003

19. Hirvensalo M, Magnussen CG, Yang X, Telama R, Heinonen I. Convergent validity of a physical activity questionnaire against objectively measured physical activity in adults: the Cardiovascular Risk in Young Finns Study. Adv Phys Educ. 2017;7:457-472. doi:10. 4236/ape.2017.74038

20. Helajärvi H, Rosenström T, Pahkala K, et al. Exploring causality between TV viewing and weight change in young and middle-aged adults. The Cardiovascular Risk in Young Finns Study. PLoS ONE. 2014;9(7):e101860. doi:10.1371/journal.pone.0101860

21. Muthén LK, Muthén BO. Mplus User's Guide (1998-2017). 8th ed. Los Angeles, CA: Muthén \& Muthén; 2017. 
22. Muthén B, Asparouhov T. LTA in Mplus: Transition Probabilities Influenced by Covariates. Mplus Web Notes: No. 13. 2011. Retrieved from http://www.statmodel.com/examples/LTAwebnote.pdf

23. Hallal PC, Andersen LB, Bull FC, et al. Global physical activity levels: surveillance progress, pitfalls, and prospects. Lancet. 2012;380(9838):247-257. PubMed ID: 22818937 doi:10.1016/ S0140-6736(12)60646-1

24. Laddu D, Rana J, Murillo R, et al. 25-year physical activity trajectories and development of subclinical coronary artery disease as measured by coronary artery calcium: the coronary artery risk development in young adults (CARDIA) study. Mayo Clin Proc. 2017;92(11):1660-1670. PubMed ID: 29050797 doi:10.1016/j. mayocp.2017.07.016

25. Barnett TA, Gauvin L, Craig CL, Katzmarzyk PT. Distinct trajectories of leisure time physical activity and predictors of trajectory class membership: a 22 year cohort study. Int J Behav Nutr Phys Act. 2008;5:57. PubMed ID: 18990250 doi:10.1186/1479-5868-5-57

26. Lounassalo I, Salin K, Kankaanpää A, et al. Distinct trajectories of physical activity and related factors during the life course in the general population: a systematic review. BMC Public Health. 2019;19:271. PubMed ID: 30841921 doi:10.1186/s12889-019-6513-y

27. Sandell L. Television viewing in Finland 2015-Finnpanel. Tennisplatsi. 2016. https://www.finnpanel.fi > lataukset > tv_year_2016. Accessed January 21, 2016.

28. McVeigh J, Smith A, Howie E, Straker L. Trajectories of television watching from childhood to early adulthood and their association with body composition and mental health outcomes in young adults. PLoS ONE. 2016;11(4):e0152879. PubMed ID: 27097324 doi:10. 1371/journal.pone.0152879

29. Burton NW, Khan A, Brown WJ, Turrell G. The association between sedentary leisure and physical activity in middle-aged adults. $\mathrm{Br} J$ Sports Med. 2012;46(10):747-752. PubMed ID: 21536709 doi:10. 1136/bjsm.2010.081430
30. Sugiyama T, Healy GN, Dunstan DW, Salmon J, Owen N. Is television viewing time a marker of a broader pattern of sedentary behavior? Ann Behav Med. 2008;35(2):245-250. PubMed ID: 18357498 doi:10.1007/s12160-008-9017-z

31. Olson JS, Hummer RA, Harris KM. Gender and health behavior clustering among U.S. young adults. Biodemography Soc Biol. 2017;63(1):3-20. PubMed ID: 28287308 doi:10.1080/19485565. 2016.1262238

32. Molanorouzi K, Khoo S, Morris T. Motives for adult participation in physical activity: type of activity, age, and gender. BMC Public Health. 2015;15(1):66. doi:10.1186/s12889-015-1429-7

33. Rovio SP, Yang X, Kankaanpää A, et al. Longitudinal physical activity trajectories from childhood to adulthood and their determinants: the Young Finns Study. Scand J Med Sci Sports. 2018;28(3):1073-1083. PubMed ID: 28981988 doi:10.1111/ sms. 12988

34. Ekelund U, Steene-Johannessen J, Brown WJ, et al. Does physical activity attenuate, or even eliminate, the detrimental association of sitting time with mortality? A harmonised meta-analysis of data from more than 1 million men and women. Lancet. 2016;388(10051):1302-1310. PubMed ID: 27475271 doi:10.1016/S0140-6736(16)30370-1

35. Dunstan DW, Salmon J, Owen N, et al. Associations of TV viewing and physical activity with the metabolic syndrome in Australian adults. Diabetologia. 2005;48(11):2254-2261. PubMed ID: 16211373 doi:10.1007/s00125-005-1963-4

36. Twisk J, Hoekstra T. Classifying developmental trajectories over time should be done with great caution: a comparison between methods. $J$ Clin Epidemiol. 2012;65(10):1078-1087. PubMed ID: 22818946 doi:10.1016/j.jclinepi.2012.04.010

37. Muthén B, Muthén LK. Integrating person-centered and variablecentered analyses: growth mixture modeling with latent trajectory classes. Alcohol Clin Exp Res. 2000;24(6):882-891. doi:10.1111/j. 1530-0277.2000.tb02070.x 